\title{
Hipopituitarismo posterior a lesión traumática cerebral
}

\section{Arturo Orrego M.}

Médico Internista Endocrinólogo, Miembro Honorario de la Asociación Colombiana de Endocrinología, Diabetes y Metabolismo

Fecha de recepción: 10/04/2016

Fecha de aceptación: 30/06/2016

\section{Introducción}

Las lesiones traumáticas cerebrales (LTC) se definen como una alteración de las funciones del cerebro u otras manifestaciones de patología cerebral causadas por fuerzas externas ${ }^{(1)}$. En la tabla 1 se esbozan los términos clínicos en relación con la severidad y fase de las LTC. Se sabe que esta entidad constituye un serio problema de salud pública en todo el mundo y que lidera como causa de muerte e invalidez ${ }^{(2)}$. Se ha calculado que $124.000(43,1 \%)$ americanos dados de alta con LTC, después de la hospitalización aguda, desarrollaron disminución de la capacidad física y mental ${ }^{(3)}$. En Europa, la suma de los casos de hospitalización por LTC y los fatales son aproximadamente de 235 por 100.000 y el porcentaje de fatalidades del $11 \%{ }^{(4)}$. Las causas más frecuentes de LTC son: accidentes por automóvil, que constituyen el 50\% de los casos, caídas, actos de violencia, los traumas causados por los deportes (natación, hockey, fútbol, fútbol americano), por deportes combativos (boxeo, kickboxing) y relacionados con accidentes por explosivos ${ }^{(5,6)}$.

Tabla 1. Términos clínicos utilizados en la LTC

\section{Severidad}

La escala de Glasgow del coma (GCS) es la más utilizada clínicamente para evaluar la severidad del trauma craneoencefálico.
Leve LTC:
GCS de 13-15
Moderado LTC: GCS de 9-12
Severo LTC: $\quad$ GCS de 3-8

\section{Fase}

Aguda: Generalmente se clasifican así a las que aparecen 10 a 14 días después del trauma

Crónica: Después de al menos tres meses de la LTC.
En 1918 se descubrió por primera vez la disfunción neuroendocrina debida a la $\operatorname{LTC}^{(7)}$, en los años que siguieron se describieron unos pocos $\operatorname{casos}^{(7)}$. Desde el año 2000 se han venido investigando las consecuencias neuroendocrinas de las LTC. Dos informes, piedras angulares en el conocimiento de la disfunción hipofisaria en la LTC, llamaron la atención sobre el compromiso hipofisario en esta entidad ${ }^{(8,9)}$, lo que motivó la investigación sobre esta asociación. La frecuencia del hipopituitarismo en la LTC varía ampliamente, entre el 15\% y el 50\%, según los métodos de laboratorio y los criterios diagnósticos utilizados $^{(10,11)}$. Antes, los estudios realizados eran de corto seguimiento. Recientemente se realizó un estudio prospectivo de cinco años de duración, en 25 pacientes $^{(12)}$. A los cinco años, la prevalencia de la deficiencia de las hormonas pituitarias anteriores eran $28 \%$, $4 \%$ y $4 \%$, de la hormona del crecimiento, las gonadotropinas y la ACTH, respectivamente ${ }^{(12)}$.

Con esta revisión breve sobre la LTC, intentamos referirnos a la epidemiología, diagnóstico, patología y a las consecuencias de las deficiencias hormonales de la pituitaria.

\section{Prevalencia de la disfunción de la hipófisis anterior postraumática en adultos}

En 2007 se realizó una revisión sistemática de la literatura y se encontró que el compromiso neuroendocrino era común después de traumas cerebrales ${ }^{(10)}$. Otros estudios pusieron en duda esta afirmación con el informe de menor frecuencia de hipopituitarismo como consecuencia del trauma cerebral ${ }^{(13,14)}$. Estudios adicionales enfatizaron sobre la necesidad de la calidad y precisión en las mediciones hormonales en la evaluación de la LTC y del compromiso hipofisario ${ }^{(13)}$.

Klose y colaboradores ${ }^{(15)}$ estudiaron la secreción de la hormona del crecimiento (HC) en 439 pacientes con trauma cerebral (aproximadamente 2,5 años después de la LTC) y 124 normales como controles, utilizando varias pruebas, cortes de normalidad y diferentes bioensayos en la medición de las hormonas. La prevalencia de deficiencia de la HC fue diferente si se utilizaban cortes de normalidad locales o los sugeridos por los autores, la administración de insulina IV u otros estímulos como la hormona liberadora de la hormona de crecimiento, más arginina o piridostigmina o dos diferentes bioensayos para medir HC. En esta investigación se encontró un significativo número de falsos positivos en los controles. En este estudio se demostró que el mejor método para estimular la 
secreción de la HC era la insulina intravenosa y que para confirmar los resultados no se debía utilizar ninguna otra prueba distinta de estímulo.

Tanriverdi y colaboradores ${ }^{(7)}$ se dieron a la tarea de efectuar una revisión sobre la prevalencia del hipopituitarismo, al menos dentro de tres meses de la LTC, motivada por los resultados tan divergentes informados.

De toda la casuística estudiada, estos autores se detuvieron preferencialmente en tres estudios con 313 pacientes, en los cuales se realizó una prueba confirmatoria después de haber obtenido un tamizaje positivo. En estos estudios encontraron deficiencias de HC en el 8,6\%; LH/FSH, 4,5\%; ACTH, 6,1\%; TSH, 1\%. El 14,7\% presentaron hipopituitarismo crónico y deficiencias hormonales múltiples en el 3,8\%. Si se hubiera usado solamente la prueba de tamizaje en los mismos 313 pacientes, la prevalencia de una única deficiencia hormonal o de múltiples deficiencias de hormonas habrían sido de $20 \%$ y $4 \%$, respectivamente.

Suponiendo, arbitrariamente que la disfunción neuroendocrina en la LTC fuera del $6 \%$ en los sobrevivientes del trauma craneoencefálico, se calcularía una incidencia de siete casos por 100.000 personas en la población por año ${ }^{(7)}$. Klose y colaboradores ${ }^{(15)}$ demostraron, sin lugar a dudas, que la deficiencia hormonal única o múltiple fue significativamente más alta en los pacientes con LTC que en los controles. La prevalencia de la deficiencia de $\mathrm{HC}$, utilizando la prueba de insulina intravenosa fue de 7,7\% en los pacientes con LTC; mientras en los controles fue sólo de 1,4\%.

En dos estudios, la prevalencia de los resultados anormales que sugerían deficiencia de ACTH en pacientes con LTC fue de $14,1 \%$ y $6,6 \%$ en los controles ${ }^{(16,17)}$.

\section{Hipopituitarismo debido a explosiones}

Recientemente se investigó la relación entre la LTC y la deficiencia pituitaria secundaria a explosiones en solda$\operatorname{dos}^{(18,19)}$. En el $42 \%$ de los participantes con lesiones por explosivos se encontró deficiencia de una o más hormonas hipofisarias. Las deficiencias hormonales más frecuentes fueron la $\mathrm{HC}$ y las FSH/LH. Los autores demostraron que el $32 \%$ de los soldados presentaron deficiencias hormonales hipofisarias que fueron más frecuentes que las halladas en controles. Estos estudios sugirieron que las lesiones por explosiones pueden producir deficiencia hipofisaria al menos 12 meses después del incidente ${ }^{(19)}$.

\section{Hipopituitarismo ocasionado por deportes causantes de traumas cefálicos repetidos}

Los deportes que incluyen contacto combativo son comunes en todo el mundo. Sólo recientemente se está llamando la atención acerca de que estos deportes pueden ser causa de LTC y que pueden llegar a producir deficiencias hipofisarias ${ }^{(20)}$.
Los deportes combativos de contacto que incluyen boxeo, kickboxing, balompié, fútbol americano y hockey sobre el hielo son los deportes que más frecuentemente pueden dar origen a traumas cerebrales crónicos. La contusión que ocurre después de los golpes directos o indirectos a la cabeza está caracterizada por el rápido compromiso, de corta duración de las funciones neurológicas que regresan a lo normal espontáneamente ${ }^{(21)}$.

Los traumas cerebrales debido a deportes pueden ser agudos o crónicos. Los agudos son similares a otros tipos de trauma encefálico cerrado como los debidos a caídas o a accidentes de tráfico. Las lesiones cerebrales crónicas incluyen contusiones recurrentes o traumas repetidos. El boxeo y el kickboxing están caracterizados por traumas crónicos, repetitivos que pueden producir LTC y deficiencia hipofisaria ${ }^{(7)}$. Se sabe que el $20 \%$ de los boxeadores profesionales pueden presentar LTC. Los golpes repetidos en la cabeza, en los boxeadores, pueden causar lesiones cerebrales, como ha sido demostrado, por alteraciones en la circulación cerebral después de peleas ${ }^{(24)}$.

\section{Deportes causantes de traumas cerebrales}

En un estudio en boxeadores aficionados en 2004 se evaluó la función pituitaria, en 11 deportistas activos o retirados, frente a controles sanos, no boxeadores. En el $45 \%$ de los individuos se encontró deficiencia de HC después de ciertos estímulos dinámicos y en muchos se halló que el factor 1 de crecimiento similar a la insulina (IGF-1) era menor en el plasma que en los controles ${ }^{(22)}$.

En 22 kickboxers aficionados, comparados con 22 controles sanos, se estudió la secreción de la hormona de crecimiento y de la ACTH, basales y con estímulos apropiados ${ }^{(25,26,27)}$. Se encontró que los kickboxers tenían una mayor incidencia de deficiencia de HC y de ACTH que los controles: 22,7\% y 9\%, respectivamente. El 27,3\% tenían como mínimo deficiencia de una de las hormonas hipofisarias.

Tanriverdi y colaboradores ${ }^{(28)}$ midieron la composición corporal en 61 boxeadores retirados o activos; 9 de los 61 eran boxeadores, $15 \%$ presentaron deficiencia de HC y el $8 \%$ presentaron deficiencia de ACTH. En otras palabras, el $47 \%$ de los retirados tuvieron deficiencia de HC. La cantidad de grasa total, la grasa abdominal, el índice de masa corporal y la circunferencia de la cadera fueron significativamente mayores en boxeadores con deficiencia de HC que en los que no la presentaron. Este estudio sugiere que la función pituitaria debe investigarse particularmente en boxeadores retirados. En otro estudio $^{(29)}$ se midieron el IGF-1 y la leptina, en boxeadores activos y en boxeadores aficionados retirados y en controles sanos, de la misma edad. El IGF-1 se encontró significativamente más bajo en el plasma en los boxeadores retirados que en los activos competitivamente y en competidores adultos. Se sugirió que los niveles bajos de IGF-1 podían ser respon- 
sables de los parámetros anormales antropométricos y de la composición del organismo. No se ha demostrado con estudios prospectivos que los boxeadores tengan mayor riesgo de enfermedad cardiovascular, a pesar de que pueden presentar deficiencia de HC.

Kelly y asociados ${ }^{(30)}$, en un estudio prospectivo de jugadores de fútbol retirados con relativa baja calidad de vida, encontraron una prevalencia de deficiencia hipofisaria y de síndrome metabólico en el $23,5 \%$ y $50 \%$, respectivamente, lo que sugirió que estos hallazgos podrían haber incidido en la pobre calidad de vida observada en estos exdeportistas.

\section{Disfunción hipofisaria y trastornos cognoscitivos en las LTC}

La disfunción cognoscitiva puede ser un problema grande después de las LTC. Las deficiencias neurocognoscitivas traen consigo un acúmulo de dificultades en las personas que tuvieron una LTC significativa, relacionadas con la atención, la memoria, el proceso en la ejecución de funciones más elaboradas como el lenguaje y las habilidades visoespaciales ${ }^{(7)}$. Tales deficiencias pueden dificultar el regreso útil, independiente de los afectados dentro de la sociedad.

Las manifestaciones cognoscitivas de las deficiencias hormonales pueden ser obvias o enmascaradas y pueden pasar como manifestaciones de la LTC y no identificarlas como propias de las deficiencias hormonales. El diagnóstico y tratamiento del hipopituitarismo postraumático puede ser esencial en la recuperación cognoscitiva causada por una lesión cerebral.

\section{Consecuencias metabólicas de LTC}

El hipopituitarismo, en general, y la deficiencia de la HC, en particular, están asociados a alteraciones metabólicas ${ }^{(7)}$, especialmente relacionadas con la glucemia, la resistencia a la insulina y la hipertrigliceridemia. En vista de los riesgos de enfermedades cardiovasculares tempranas y muerte en los pacientes con hipopituitarismo, predominantemente con deficiencia de hormona del crecimiento, se debe prestar atención a los pacientes portadores de trauma cerebral asociado a deficiencias hormonales hipofisarias.

\section{Patofisiología del hipopituitarismo causado por LTC}

Las causas del hipopituitarismo posterior a trauma no se conocen plenamente. Se ha sugerido la interacción de múltiples mecanismos, muchos no demostrados, ni estudiados completamente en animales de experimentación o en humanos. Cualquier clasificación del daño cerebral debido a traumas cefálicos debe tener en cuenta el espectro completo de la presentación clínica y el desenlace.
Para mejorar el desenlace final es necesaria la comprensión de los factores celulares, bioquímicos y moleculares comprometidos en las LTC. Hasta el momento no existe un consenso bioquímico y neuropatológico que clarifique y haga posible una clasificación acertada sobre las LTC, utilizando los hechos clínicos y patológicos ${ }^{(7)}$.

Los mecanismos biomédicos más importantes comprometidos en la LTC incluyen: 1) daño focal del sistema nervioso por trauma por contacto, con fracturas del cráneo, contusiones, laceraciones, hemorragia intracraneana, hematomas intracerebrales; o 2) daño difuso, multifocal debido a la aceleración y desaceleración con daño cerebral, por contusión, lesión difusa axonal, compromiso vascular, daño isquémico e hipóxico y edema cerebral ${ }^{(31,31)}$. Algunos clasifican la LTC en aguda y crónica: 1) Las lesiones agudas que comprenden las LTC leves o contusiones moderadas, pueden llevar a la muerte y, 2) las lesiones cerebrales crónicas, llamadas encefalopatía crónica traumática, que es un trastorno neurodegenerativo debido a traumas cerebrales crónicos, repetitivos, especialmente en atletas que participan en deportes de contacto ${ }^{(7)}$.

Por definición, la contusión es una síndrome clínico en el que existe un compromiso inmediato y transitorio del sistema nervioso central o mental, caracterizado por trastornos de la visión o alteraciones de la conciencia o por otros signos debido al compromiso de otras regiones cerebrales, secundarias a afectación de los axones, por compresión, con disrupción de la fibras axonales y la mielina, causadas por las fuerzas de aceleración y desaceleración producidas por el trauma ${ }^{(31,32)}$. La TAC o la resonancia magnética convencionales no hacen diagnóstico del compromiso axonal.

La evolución de la LTC está determinada por los estados diferentes que se deben a mecanismos distintos. La lesión primaria se presenta en el momento del impacto. El segundo insulto, el daño secundario no mecánico, se caracteriza por procesos neuropatológicos, iniciados en el momento del trauma con manifestaciones clínicas tardías, en las cuales participan ciertos hechos, hipertensión intercraneana, hipoxia, hiponatremia e isquemia cerebral ${ }^{(7)}$.

El estado inicial del trauma cerebral está caracterizado por daño directo del tejido nervioso y compromiso de la regulación del flujo sanguíneo cerebral (hipo o hiperfusión). La isquemia cerebral se debe a distorsión de los vasos sanguíneos debida a desplazamiento mecánico, a hipotensión por falta de la autorregulación, a inadecuada suplencia de neurotransmisores $\mathrm{u}$ óxido nítrico y por potenciación de la vasoconstricción inducida por prostaglandinas ${ }^{(31,32)}$. El vasoespasmo postraumático es un factor secundario importante que agrava la isquemia e hipoxia. Como consecuencia de la isquemia cerebral e hipoxia que produce el trauma cerebral se acumula ácido láctico, debido a la glucólisis anaerobia, lo que aumenta la permeabilidad de las membranas con formación del edema cerebral, el cual aumenta la presión intracerebral, con aumento de la isquemia ${ }^{(33,34)}$. 
El segundo estado de la cascada fisiopatológica se caracteriza por despolarización de las membranas, acompañada de una excesiva liberación de neurotransmisores, tales como el glutamato, el aspartato, con la consecuente apertura de los canales $\left(\mathrm{Na}^{+}, \mathrm{K+}, \mathrm{Ca}^{2+}\right)^{(7)}$. El estrés oxidativo que ocurre con el trauma cerebral contribuye al daño microvascular y neural. La LTC produce respuestas inflamatorias e inmunológicas en el tejido cerebral. Tanto los insultos primarios como los secundarios pueden activar la liberación de mediadores proinflamatorios (citocinas y otros factores). Los procesos descritos pueden llevar a la degradación de la membrana celular y estructuras vasculares y eventualmente llevar a necrosis o apoptosis de las neuronas ${ }^{(7,33,34)}$.

\section{Hipótesis sobre el hipopituitarismo debido a la LTC}

Debido a la localización anatómica de la glándula pituitaria, el tallo, el hipotálamo y a que los vasos largos del sistema porta pasan a través del diafragma de la silla, estas estructuras son muy vulnerables a traumas mecánicos directos, a cambios isquémicos y a compresión por edema cerebral ${ }^{(7,35,36,37)}$. Como resultado del primer impacto traumático puede haber daño mecánico caracterizado por edema, fractura craneana, hematoma, lesiones por desplazamiento y desgarro del hipotálamo y de los vasos del sistema porta. En los casos leves de LTC debidos a deportes de contacto y a traumas craneanos crónicamente repetidos, las contusiones con disrupción de los axones, originadas en fuerzas de desplazamiento y ruptura pueden afectar las funciones hipotálamo-hipofisarias. También se han encontrado cambios microestructurales y microvasculares, especialmente en la LTC leve ${ }^{(7)}$. Se han definido cambios secundarios derivados del primer trauma que pueden contribuir a la prolongación de la primera lesión y a profundizar el daño vascular y pituitario ${ }^{(31,32)}$. Entre estos hechos están el edema cerebral $^{(33)}$, la liberación de citocinas, la disminución del flujo vascular, la presión arterial alta, la hipotensión, el edema, la hiponatremia, la isquemia cerebral e hipoxia, el aumento de radicales libres, el estrés oxidativo, la disfunción de las neuronas y el aumento de los neurotransmisores. Es entonces la hipótesis vascular la más creíble en la patogénesis del hipopituitarismo crónico después de la LTC $^{(35)}$. Sin embargo, los cambios dinámicos hormonales como los observados en las LTC, la recuperación de la secreción hormonal con la posibilidad de nuevo compromiso posterior de la secreción de las hormonas hipofisarias, no es fácilmente explicable con la hipótesis vascular. Más recientemente se han invocado como causas patogenéticas de la LTC, la autoinmunidad y factores genéti$\cos ^{(11,38,39)}$. El trauma craneoencefálico puede disparar una cascada de eventos vasculares e histopatológicos que conducen a neuroinflamación, a la secreción de citocinas, interleucinas, y a la formación de anticuerpos dirigidos contra el sistema ner- vioso. Títulos altos de anticuerpos pueden dañar la barrera hematoencefálica y los tanicitos e intensificar los efectos neuroinflamatorios. Todavía se discute la probable existencia de factores neuroprotectores (la capacidad de regeneración de los vasos portales y de las células stem hipofisarias en la recuperación de las funciones pituitarias ${ }^{(7)}$.

Se sugiere que el hipopituitarismo crónico secundario a la LTC pudiera ser consecuencia de un balance dinámico entre mecanismos neurodegenerativos y neuroprotectores ${ }^{(7)}$. Se necesita mucha investigación experimental en animales que contemple todos los diferentes grados de intensidad y de presentación clínica para tratar de comprender la fisiopatología del hipopituitarismo secundario a la LTC y de crear medios para su prevención y tratamiento ${ }^{(7)}$.

\section{Diagnóstico y tratamiento del hipopituitarismo}

En la figura 1 se muestra el tamizaje prospectivo del hipopituitarismo diagnosticado después de un trauma craneoencefálico leve. En la figura 2 se detalla el tamizaje prospectivo

Figura 1. Tamizaje para estudio de la función pituitaria anterior después de una LTC leve complicada. La evaluación al año postrauma cerebral necesita mayor estudio y confirmación

Pacientes con LTC leve complicada

Investigue la deficiencia de $\mathrm{ACTH}$, con cortisol basal en ayunas (en los días 1-4 después de la LTC); busque hiponatremia, hipotensión, etc. Investigue deficiencia de ACTH antes de dar salida al paciente.

Trate la deficiencia de ACTH

Ordene a los 6 meses, estudio basal y dinámico de ACTH

Trate las deficiencias de $\mathrm{ACTH}, \mathrm{TSH}$ y $\mathrm{FSH} / \mathrm{LH}$

A los 12 meses:

- Nueva evaluación clínica

- Medición hormonal basal y pruebas dinámicas para HC y ACTH, en busca de deficiencias.



Cada año durante 5 años: Evalué mediante clínica y laboratorio para hipopituitarismo; las deficiencias de hormonas hipofisarias pueden reaparecer más tarde
Evalúe cada año por 5 años (la deficiencia pituitaria puede recuperarse) Evaluación clínica y de laboratorio con pruebas dinámicas para deficiencias hipofisaria de ACTH y HC, si está indicado 
Figura 2. Tamizaje de las funciones de la pituitaria anterior postrauma cerebral en los casos con LTC moderada o severa

Pacientes con LTC moderada o severa

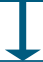

Investigue la deficiencia de ACTH basal, midiendo cortisol, en los días 1 a 10 postrauma, especialmente si existe hiponatremia, hipotensión arterial o hipoglucemia.

Evalúe deficiencia de ACTH, antes de dar de alta al paciente.

Trate la deficiencia de ACTH

Ordene a los 6 meses, medición basal de hormonas y pruebas dinámicas para deficiencia de ACTH

Trate las deficiencias de ACTH, $\mathrm{TSH}$ y $\mathrm{FSH} / \mathrm{LH}$ como se requiere

Evalue a los 12 meses:

- Evaluación clínica

Medición de hormonas basales y pruebas dinámicas para deficiencia de ACTH y HC.

\begin{tabular}{|l|l|}
\hline $\begin{array}{l}\text { No neficiencia } \\
\text { hormonal }\end{array}$ & \multicolumn{1}{|l|}{$\begin{array}{l}\text { Deficiencia de una } \\
\text { o varias hormona }\end{array}$} \\
\hline $\begin{array}{l}\text { Esté atento a signos y síntomas } \\
\text { de deficiencia de hormonas } \\
\text { pituitarias }\end{array}$ & $\begin{array}{l}\text { El paciente debe evaluarse } \\
\text { mediante clínica y por } \\
\text { laboratorio, igual que otros } \\
\text { tipos de hipopituitarismo } \\
\text { (recuperación hormonal de la } \\
\text { deficiencia es rarísima) }\end{array}$ \\
\hline
\end{tabular}

de la función hipofisaria anterior en los casos de LTC moderada o extrema.

En la fase aguda de la LTC, que comprende los primeros días después del trauma cerebral, entre 10 y 14 días, no existe evidencia clara que se deba investigar o dar remplazo hormonal de las tropinas deficitarias la HC, la FSH/lH y la TSH. No se debe olvidar estudiar en la fase aguda la secreción de ACTH, en condiciones basales y posestímulo, porque la deficiencia de esta tropina puede producir la muerte, si no se trata oportunamente.

En el hipopituitarismo postraumático crónico (1 a 3 meses después del trauma cerebral), la deficiencia de las hormonas hipofisiarias se trata con las dosis adecuadas de remplazo como se procede en otros casos de hipopituitarismo parcial o total no traumático. Se debe prestar suma atención a la deficiencia de la hormona del crecimiento porque en el adulto puede ser problemática y disminuir el estilo de vida. La defi- ciencia de esta hormona en adultos puede producir trastornos de la glucemia, resistencia a la insulina, síndrome metabólico, obesidad centrípeta, déficit cognoscitivo, hipertrigliceridemia y compromiso muscular ${ }^{(7)}$.

\section{Resumen}

Las LTC son una de las causas más comunes de hipopituitarismo en el adulto. La deficiencia hipofisaria postraumática se descubrió hace más de 90 años, pero sólo últimamente se le ha prestado la atención que merece. Los síntomas del hipopituitarismo mínimo son lentamente progresivos y no específicos, lo que dificulta su diagnóstico y retarda el tratamiento. Las causas más comunes de las LTC que producen deficiencia hipofisaria son los accidentes de tránsito y las caídas. El boxeo y el kickboxing, que característicamente producen traumas cerebrales repetidos, son causas del LTC, recientemente estudiados. El estallido de sustancias explosivas puede ser causa potencial de deficiencia hipofisaria anterior. La fisiopatología del hipopituitarismo postraumático cerebral no se conoce completamente. La predisposición genética, la autoinmunidad y la neuroinflamación se han propuesto como factores responsables del desarrollo de la deficiencia hipofisaria anterior. La formación de anticuerpos contra la pituitaria en alta concentración puede influir en el daño hipofisario a largo plazo. La neuroinflamación persistente producida por el trauma craneoencefálico puede influir en la formación del hipopituitarismo en pacientes predispuestos genéticamente (portadores de APOE3/E3). En adición, la autoinmunidad puede acrecentar los efectos nocivos de la neuroinflamación, lo que podría disminuir el volumen hipofisario y la disminución de la secreción de las tropinas. La deficiencia de HC es más frecuente en los portadores de las LTC y en los deportes combativos como el boxeo. El hipopituitarismo en personas con LTC puede acompañarse de hipófisis pequeñas demostradas por la resonancia magnética, lo que sugiere un mecanismo isquémico. Los pacientes con LTC y deficiencia hipofisaria anterior con frecuencia presentan trastornos metabólicos en particular demostrados en los niveles de glucosa, la resistencia a la insulina e hipertrigliceridemia, traducidos en una disminución en la calidad de vida.

La comprensión de posibles mecanismos neuroprotectores (capacidad de los vasos portales de regenerarse y la activación de las células progenitoras stem capaces de recuperar hipotéticamente las funciones adenohipofisiarias), es necesaria para tratar de prevenir el desarrollo o del hipopituitarismo permanente. 


\section{Referencias}

1. Menon DK, Schwab K, Wright DW, et al. Position statement: definition of traumatic brain injury. Arch Phys Med Rehabil. 2010; 91:1637-1640.

2. Corrigan JD, Selassie AW, Orman JA. The epidemiology of traumatic brain injury. Head Trauma Rehabil. 2010; 25:72-80.

3. Selassie AW, Zaloshnja E, Langlois JA, et al. Incidence of long-term disability following traumatic brain injury hospitalization. United States. 2003. J Head Trauma Rehabil. 2008; 23:123-131.

4. Tagliaferny F, Compagnone C, Korsic M,et al. A systematic review of brain injury epidemiology in Europe. ActaNeurochir (wien). 2006; 148:255-268.

5. Mckinlay A, Grace RC, Horwood LJ, et al. Prevalence of traumatic brain in jury among children, adolescents and young adults: prospective evidence from a birth control. Brain Inj. 2008; 22:175-181.

6. Consensus conference. Rehabilitation of persons with traumatic brain injury. NIH consensus Development Panel on Rehabilitation of Persons with Traumatic Brain Injury. JAMA. 1999; 282:974-983.

7. Tanriverdi F,Schneider JA, Gianluca A, et al. Pituitary dysfunction after traumatic brain injury. A clinical and pathophysiological approach. Endocrine Review. 2015;36: 305-342.

8. Benvenga S,Campenni A, Ruggeri RM, et al . Clinical review 113: hipopituitarism secondary to head trauma. J Clin Endocrinol Metab. 200;85:13531361.

9. Lieberman SA, Oberoi AL, Gilkison CR, et al. Prevalence of neuroendocrine disfunction en patients recovering from traumatic brain injury. J Clin Endocrinol Metab. 2001;86: 2752-2756.

10. Schneider HJ, Kreitschmann- Andermohr I, Ghigo E, et al. Hypothalamopituitary disfunction following, traumatic brain injury and aneurysmal subarachnoid hemorrhage: a systematic review. JAMA. 2007;298: 1429-1483

11. Tanriverdi F, Senyurek H, Unluhizarci K, et al. High risk of hypopituitarism after traumatic brain injury: a prospective investigation of anterior pituitary function in the acute phase and 12 months after trauma. J Clin Endocrinol Metb. 2006;91:2105- 2111.

12. Tanriverdi f, De Bellis A, Ulutabanca $\mathrm{H}$, et al. A five years prospective investigation of anterior pituitary function after traumatic brain injury: is hypopituirarism long- term after head trauma associated with autoimmunity?. J Neurotrauma. 2013;30:1426-1433.

13. Kokshoorn NE, Wassenaar MJ, Biermasz NR, et al. Hipopituitarism following traumatic brain injury: prevalence is affected by the use of different dynamic tests and different normal values. Eur J Endocrinol.2010; 162: 11-18.

14. Bavisetty NE, Bavisetty S, Mc Artur DL, et al. Chronic hipopituitarysm after traumatic brain injury: risk assessment and relationship to outcome. Neurosurgery. 2008;62:1080-1093; discussion 1093-1094.

15. Klose M, Stochholm K, Jamukonte J, et al. Prevalence of posttraumatic growth hormone deficiency highly dependent on the diagnostic setup: results from the Danish National Study on Posttraumatic Hypopituitarism. J Clin Endocrinol Metab. 2014;99:101-110.

16. Agha A, Rogers B, Sherlock M, et al. Anterior pituitary disfunction in survivors of traumatic brain injury. J Clin Endocrinol Metab. 2004; 89:49294936.

17. Klose M,Juul A, Poulsgaard L,et al. Prevalence and predictive factor of posttraumatic hypopituitarism. ClinEndocrinol (Oxford).2007; 67:193-201.

18. Wilkinson CW, Pagulayan KF,Petrie EC, et al . High prevalence of chronic pituitary and target-organ hormone abnormalities after blast- related mild traumatic brain injury. Front Neurol. 2013; 74:527-536.

19. Baxter D, Sharp DJ, Freeney C. et al. Pituitary disfunction after blast traumatic brain injury. Ann Neurol. 2013;74: 527-536.

20. Theadom A, Starkey NJ, Dowell T, et al. Sports related brain injury in the general population: an epidemiological study. J Sci Med Sport. 2014;17:591596.

21. Aubry M, Cantu R, Dvorak J, et al. Summary and agreement statement of the First International Conference on Concussion Sport, Vienna 2001. Recommendations for the improvement of safety and health of athletics who may suffer concussive injuries. Br J Sports Med. 2001;36:6-10.

22. Kelestimur F, Tanriverdi F, Atmaka H, et al. Boxing as a sport activity associated with isolated GH deficiency. J Endocrinol Invest. 2004;27:RC28-RC-32.

23. Jordan BD, Chromic traumatic brain injury associated with boxing. Semin Neurol. 2000;20:179-185.

24. Zetterberg H, Tanriverdi F, Unluhizarci K, et al. Sustained release of neuronspecific-enolase to serum in amateurs boxers. Brain Inj.2009;23:723-726.

25. Tanriverdi FUnluhizarci K, Cokservin B, et al. Kickboxing sport as a new cause of traumatic brain injury-mediated hypopituitarism.Clin Endocrinol (Oxford). 2007; 66:360-366

26. Gartland S, Malik MH, Lovell ME, et al. Injury and injury rates in Muay Thaikick boxing. Br J Sports Med. 2001; 35:308-317.

27. Zagryn TR, Finch C F, McCrory P, et al. A 16 year study of injuries to professional kickboxers in the state of Victoria, Australia. Br J Sports Med. 2003 37:448-451.

28. Tanriverdi k, Kocyigit I, Unluhizarci K, et al . Brief communication: Pituitary volume and function in competing and retired male boxers. Ann Intern Med. 2008;148:827-831.

29. Tanriverdi K, K Kocyigit, Unluhizarcy K,et al. Body composition, serum IGF1 and leptin levels changes in amateur boxers: retired boxers have risk factor for cardiovascular disorders. Obes Metab . 2008; 4:118-122.

30. Kelly DF, Chaloner C, Evans D, et al. Prevalence of pituitary hormone disfunction, metabolic syndrome and impaired quality of life in retired professional football players : a prospective study. J Neurotraums. 2014; 31:11611171.

31. Grahm DI, McInTosh TK, Maxwell WI, et al. Recent advances in neurotrauma, J Neuropath Exp Neurol. 2000; 59:641-651.

32. Nortje J, Menon Dk. Traumatic brain injury : physiology, mechanism, and outcome. Curr Opin Neurol. 2004; 17:711-718.

33. Marmarou A, Fatouros PP, Barzo P, et al. Contribution of edema and cerebral blood volume to traumatic swelling in head-injuried patients. J Neurosurg. 200; 93:183-193.

34. Marmarou A, Signoretti S, Fatouros PP, et al. Predominance of cellular edema in traumatic brain. J Neurosurg. 2006; 104:720-730.

35. Beahn LA, Phillis J, Thompson CJ, et al. Neuroendocrine disorders afte traumatic brain injury. J Neurol Neurosurg Psychiatry. 2008,79:753-759.

36. Daniel PM, Prichard MM, Treip CS. Traumatic Infarction of the anterior lobe of the pituitary gland. Lancet. 1959; 2:927-931.

37. Yuan XQ, Wade CE. Neuroendocrine abnormalities in patients with traumatic brain injury. Front Neuroendocrinol. 1991; 12:209-230.

38. Tanriverdi $F$, Ulutabanca $H$, Unluhizarci $K$, et al. Three years prospective investigation of anterior pituitary function after traumatic brain injury: a pilot study. Clin Endocrinol (Oxford). 2008;68:573-579. 